\title{
Genetic determinants of clinical phenotype in hypertrophic cardiomyopathy
}

\author{
Lazar Velicki ${ }^{1,2^{*}+}$, Djordje G. Jakovljevic ${ }^{3,9^{*}+}$, Andrej Preveden ${ }^{1,2}$, Miodrag Golubovic ${ }^{1,2}$, Marija Bjelobrk ${ }^{1,2}$,
} Aleksandra llic ${ }^{1,2}$, Snezana Stojsic ${ }^{1,2}$, Fausto Barlocco ${ }^{4}$, Maria Tafelmeier $^{5}$, Nduka Okwose ${ }^{3}$, Milorad Tesic ${ }^{6}$, Paul Brennan ${ }^{3}$, Dejana Popovic ${ }^{6}$, Arsen Ristic ${ }^{6}$, Guy A. MacGowan ${ }^{3}$, Nenad Filipovic ${ }^{7,8}$, Lars S. Maier ${ }^{5 \dagger}$ and lacopo Olivotto ${ }^{4 \dagger}$

\begin{abstract}
Background: Hypertrophic cardiomyopathy $(\mathrm{HCM})$ is the most common inherited cardiovascular disease that affects approximately one in 500 people. HCM is a recognized genetic disorder most often caused by mutations involving myosin-binding protein C (MYBPC3) and $\beta$-myosin heavy chain (MYH7) which are responsible for approximately three-quarters of the identified mutations.

Methods: As a part of the international multidisciplinary SILICOFCM project (www.silicofcm.eu) the present study evaluated the association between underlying genetic mutations and clinical phenotype in patients with HCM. Only patients with confirmed single pathogenic mutations in either MYBPC3 or MYH7 genes were included in the study and divided into two groups accordingly. The MYBPC3 group was comprised of 48 patients (76\%), while the MYH7 group included 15 patients (24\%). Each patient underwent clinical examination and echocardiography.

Results: The most prevalent symptom in patients with MYBPC3 was dyspnea (44\%), whereas in patients with MYH7 it was palpitations (33\%). The MYBPC3 group had a significantly higher number of patients with a positive family history of HCM ( $46 \%$ vs. $7 \% ; p=0.014)$. There was a numerically higher prevalence of atrial fibrillation in the MYH7 group ( $60 \%$ vs. $35 \%, p=0.085)$. Laboratory analyses revealed normal levels of creatinine $(85.5 \pm 18.3 \mathrm{vs.} 81.3 \pm 16.4 \mu \mathrm{mol} / \mathrm{l}$; $p=0.487)$ and blood urea nitrogen ( $10.2 \pm 15.6 \mathrm{vs} .6 .9 \pm 3.9 \mathrm{mmol} / ; ; p=0.472)$ which were similar in both groups. The systolic anterior motion presence was significantly more frequent in patients carrying MYH7 mutation (33\% vs. 10\%; $p=0.025$ ), as well as mitral leaflet abnormalities ( $40 \%$ vs. $19 \% ; p=0.039$ ). Calcifications of mitral annulus were registered only in MYH7 patients ( $20 \%$ vs. $0 \% ; p=0.001)$. The difference in diastolic function, i.e. E/e' ratio between the two groups was also noted (MYBPC3 8.8 \pm 3.3, MYH7 13.9 $\pm 6.9, p=0.079$ ).
\end{abstract}

Conclusions: Major findings of the present study corroborate the notion that MYH7 gene mutation patients are presented with more pronounced disease severity than those with MYBPC3.

Keywords: Hypertrophic cardiomyopathy, HCM, Hereditary cardiac disease, Left ventricular hypertrophy, MYBPC3, MYH7

\footnotetext{
*Correspondence: lazar.velicki@mf.uns.ac.rs;

djordje.jakovljevic@newcastle.ac.uk

†Lars S. Maier and Olacopo Olivotto: Joint last authors

${ }^{\dagger}$ Lazar Velicki and Djordje G. Jakovljevic: Joint first authors

${ }^{1}$ Faculty of Medicine, University of Novi Sad, Novi Sad, Serbia

${ }^{3}$ Cardiovascular Research, Translational and Clinical Research Institute,

Medicine, Newcastle University, Newcastle upon Tyne Hospitals NHS

Foundation Trust, Newcastle upon Tyne, UK

Full list of author information is available at the end of the article
} 


\section{Background}

Hypertrophic cardiomyopathy ( $\mathrm{HCM})$ is the most frequent inherited disease of the myocardium, with a prevalence of approximately $0.2 \%[1,2]$. Despite the significant developments in diagnostic tools and genetic tests, the diagnosis of HCM is often delayed [2].

HCM is characterized by left ventricular (LV) hypertrophy without dilatation, in the absence of any other cardiac, systemic, metabolic, or syndromic disease that could explain myocardial hypertrophy [2-5]. Clinical presentation of HCM varies from completely asymptomatic with normal life expectancy, to typical symptoms like chest pain, shortness of breath, heart failure, palpitations, syncope, and in the worst case even sudden cardiac death $[2,6]$. Complications of non-obstructive HCM include advanced myocardial fibrosis, microvascular ischemia, and deterioration of cardiac function [7].

$\mathrm{HCM}$ is a recognized genetic disorder transmitted in an autosomal dominant fashion, caused by a single mutation in one of the sarcomeric protein genes, which can be present in either thick- or thin-filament genes $[8,9]$. The two most common mutations involving thick filament are myosin-binding protein $\mathrm{C}$ (MYBPC3) and $\beta$-myosin heavy chain (MYH7) gene mutations, which are responsible for approximately three-quarters of the identified mutations in HCM patients $[9,10]$. Aside from these two, a few other less frequent gene mutations (e.g. troponin I type 3 [TNNI3], troponin T type 2 [TNNT2], $\alpha$-tropomyosin [TPM1], $\alpha$-actin [ACTC]) are possible causes of HCM as well and are therefore also included in the routine HCM genetic testing [11]. Technological progress has made it possible to identify new genes associated with $\mathrm{HCM}$-numerous other genes that do not encode sarcomere proteins but rather genes encoding the synthesis of Z-disk proteins and proteins involved in the calcium signaling pathway. With the introduction and implementation of the next-generation sequencing solutions, the identification of nearly 50 gene mutations associated with some form of HCM throughout literature has become possible [12].

Regardless of the mutation type, the same pathophysiology mechanisms are responsible for the development of typical HCM phenotype and disease progression. Disrupted sarcomere properties due to the mutations cause impaired relaxation and lead to diastolic dysfunction, which is followed by hyperdynamic contractility and hypertrophy of the LV in the later course $[9,11]$.

Due to variable penetrance and expressivity, the phenotypic characteristics of $\mathrm{HCM}$ are multifaceted and may be influenced by other factors beyond single pathogenic mutations [13]. In addition to LV hypertrophy, phenotypic HCM expression also includes myocardial hypercontractivity, myofibril disorganization, fibrosis, as well as the presence of mild myocardial inflammation. Although the clinical phenotype can partially differ depending on the affected gene, no distinctive correlation between disease severity and specific genes has been established. Moreover, clinical features such as disease penetration, hypertrophy severity, and patient prognosis are known to vary depending on different mutations within the same gene [11].

The precise link between determined underlying gene mutation and the clinical course remains elusive in this heterogeneous condition. The motivation to compile this HCM patient registry was to try to define what patient features are more prevalent with specific gene mutations and to establish whether the level of disease expression might be linked to one of the two most common mutations responsible for HCM. The goal was to reveal and distinguish subtle differences that may exist in clinical presentation and, more importantly, in heart structure and function recorded by cardiac imaging (i.e. echocardiography) between different gene mutations, thus providing essential information for the computational model development. Moreover, data from this study will also complement the clinical trial (NCT03832660 at clinicaltrials.gov) evaluating the effects of pharmacological (sacubitril/valsartan) versus lifestyle intervention in HCM patients [14], also a fundamental part of the SILICOFCM project.

\section{Methods}

As a part of the international multidisciplinary SILICOFCM project (www.silicofcm.eu) developing a computational platform for in silico clinical trials of familial cardiomyopathies, the present study evaluated the association between genetic mutations and clinical phenotype in patients with HCM. The study protocol was approved by the UK National Health Service Health Research Authority North East-Tyne \& Wear South Research Ethics Committee with the reference number 18/NE/0318 and was adopted by the Institutional Review Board of each participating center. All patients provided written informed consent and all procedures were conducted following the Declaration of Helsinki.

\section{Study design}

Participating centers included patients with diagnoses of HCM who were identified in the period from June 2018 to February 2019.

The diagnosis of HCM was defined according to the European Society of Cardiology guidelines i.e. maximal LV wall thickness of $\geq 15 \mathrm{~mm}$ on echocardiography, in the absence of any other cardiac or systemic disease that would be capable of producing myocardial hypertrophy, 
such as afterload abnormalities like aortic valve stenosis or arterial hypertension [5]. Of the total of $74 \mathrm{HCM}$ patients, 11 were excluded because of the relatively small number of other gene mutations (TNNI3-5 patients, TNNT2-2 patients, TPM1-1 patient, myosin heavy chain 6-1 patient, myosin light chain 2-1 patient, lamin $\mathrm{A} / \mathrm{C}-1$ patient) might have biased the overall study analysis. In the final analysis, the study included a total of 63 adult patients with a confirmed diagnosis of HCM.

We excluded patients with significant atherosclerotic coronary artery disease $(>50 \%$ stenosis in a major artery), patients with prior cardiac surgery (including septal myectomy), alcohol septal ablation, major LV outflow obstruction with pressure gradient $>50 \mathrm{mmHg}$, and chronic renal failure $\left(<30 \mathrm{ml} / \mathrm{min} / 1.73 \mathrm{~m}^{2}\right)$.

\section{Genetic testing}

Genetic testing was performed from peripheral blood samples acquired by phlebotomy with the utilization of the QIAamp DNA Blood BioRobot MDx kit (QIAGEN $\mathrm{GmbH}$, Hilden, Germany). Polymerase chain reaction with primers was used for the amplification of candidate exons.

Blood samples were analyzed for the presence of the 8 most common mutations, which represent the basis of the commonly available genetic tests for HCM. These mutations include the protein-coding exons responsible for encoding myosin-binding protein C (MYBPC3), thick-filament proteins ( $\beta$-myosin heavy chain [MYH7] and the regulatory and essential light chains [MYL2 and MYL3]), and thin-filament proteins (troponin $\mathrm{T}$ type 2 [TNNT2], troponin I type 3 [TNNI3], $\alpha$-tropomyosin [TPM1], and $\alpha$-actin [ACTC]).

Only patients with confirmed single pathogenic mutations in either MYBPC3 or MYH7 genes were included in the study. Based on the identified gene mutation the patients were divided into two groups. The MYBPC3 group was comprised of 48 patients (76\%), while the MYH7 group included 15 patients (24\%).

\section{Electrocardiogram and ECG Holter monitoring}

The ECG was performed using a standard 12-leadelectrocardiogram in a supine position. To identify sporadic arrhythmia, all participants were asked to wear an ECG-Holter monitor for $24 \mathrm{~h}$ and to keep a diary of activities and symptoms.

\section{Echocardiography}

Transthoracic echocardiography was performed in all patients. Images were obtained using regular parasternal and apical views. All the parameters were calculated and indexed for body surface area (BSA).
LV wall thickness and chamber dimensions were measured using the parasternal long-axis view $[14,15]$. The Devereux formula [16] was used to calculate LV myocardial mass.

LV geometry was assessed by the relative wall thickness which is calculated as two times the LV posterior wall thickness divided by LV end-diastolic diameter.

LV systolic and diastolic volumes were measured with Simpson's modified biplane method using apical 4-chamber and 2-chamber views, and LV systolic function was expressed through the ejection fraction [15].

For diastolic function assessment, an apical 4-chamber view was used [17]. Blood flow through the mitral valve was measured by pulsed-wave Doppler between the tips of mitral leaflets and the peak modal velocity in early diastole (E) was determined. Velocities of basal regions at lateral and septal mitral annulus were recorded using tissue Doppler imaging and then their average ratio $\left(\mathrm{e}^{\prime}\right)$ was computed.

The filling pressure of the LV was expressed through the $\mathrm{E} / \mathrm{e}^{\prime}$ ratio, which is the most accurate indicator of diastolic function according to the literature [18].

\section{Statistical analysis}

Continuous variables are expressed as mean values \pm standard deviation and categorical variables are presented as absolute numbers and percentages. Quantitative data distribution was assessed using the Kolmogorov-Smirnov test. Mean values of continuous variables were compared using the independent samples t-test or Mann-Whitney $U$ test, whereas categorical variables were compared using the chi-square test. Statistical significance for all tests was set at the $p$ value of $<0.05$. All the analyses were done in SPSS version 20.0.

\section{Results}

The mean age of HCM patients regardless of genetic mutation was $51.1 \pm 14.2$ years and most of them were male 48 (76\%). They were slightly overweight according to their mean BMI of $26.4 \pm 4.4 \mathrm{~kg} / \mathrm{m}^{2}$. One-third of patients $(36 \%)$ had a positive family history for HCM.

Differences in terms of patient profile depending on genetic mutation are shown in Table 1.

There was no significant difference between patients carrying the MYBPC3 and MYH7 mutations regarding age ( $49.8 \pm 14.3$ vs. $55.1 \pm 13.3$ years, $p=0.211)$ and gender distribution ( $21 \%$ vs. $33 \%$ females, $p=0.321$ ).

The most prevalent symptom in patients with MYBPC3 was dyspnea (44\%), whereas in patients with MYH7 it was palpitations (33\%). Other less frequently reported symptoms included fatigue, chest pain, and syncope, with similar distribution among the groups. 
Table 1 General characteristics of patients with MYBPC3 and MYH7 gene mutation

\begin{tabular}{|c|c|c|c|c|}
\hline & Overall & MYBPC3 & MYH7 & $p$ value \\
\hline Age (years) & $51.1 \pm 14.2$ & $49.8 \pm 14.3$ & $55.1 \pm 13.3$ & 0.211 \\
\hline Females, $n(\%)$ & $15(23.8 \%)$ & $10(20.8 \%)$ & $5(33.3 \%)$ & 0.321 \\
\hline BMI $\left(\mathrm{kg} / \mathrm{m}^{2}\right)$ & $26.4 \pm 4.4$ & $26.1 \pm 4.6$ & $27.8 \pm 3.1$ & 0.260 \\
\hline Fatigue, $n(\%)$ & $9(14.3 \%)$ & $7(14.6 \%)$ & $2(13.3 \%)$ & 0.881 \\
\hline Dyspnea, $n(\%)$ & $25(39.7 \%)$ & $21(43.7 \%)$ & $4(26.7 \%)$ & 0.238 \\
\hline Chest pain, $n(\%)$ & $6(9.5 \%)$ & $4(8.3 \%)$ & $2(13.3 \%)$ & 0.565 \\
\hline Palpitations, n (\%) & $13(20.6 \%)$ & $8(16.7 \%)$ & $5(33.3 \%)$ & 0.177 \\
\hline Syncope, $n(\%)$ & $10(15.9 \%)$ & $9(18.7 \%)$ & $1(6.6 \%)$ & 0.264 \\
\hline Family history of HCM, $n(\%)$ & $23(36.5 \%)$ & $22(45.8 \%)$ & $1(6.6 \%)$ & $0.014^{*}$ \\
\hline \multicolumn{5}{|l|}{ Comorbidities } \\
\hline Diabetes mellitus, n (\%) & $3(4.8 \%)$ & $3(6.2 \%)$ & - & - \\
\hline $\begin{array}{l}\text { Chronic obstructive pulmonary disease, } \\
n(\%)\end{array}$ & $2(3.2 \%)$ & $2(4.2 \%)$ & - & - \\
\hline Thyroid dysfunction, $n$ (\%) & $8(12.7 \%)$ & $7(14.6 \%)$ & $1(6.7 \%)$ & 0.422 \\
\hline Anemia, $n(\%)$ & $1(1.6 \%)$ & $1(2.1 \%)$ & - & - \\
\hline \multicolumn{5}{|l|}{ Laboratory analyses } \\
\hline Glucose (mmol/l) & $5.6 \pm 1.2$ & $5.8 \pm 1.3$ & $5.0 \pm 0.6$ & 0.071 \\
\hline Creatinine $(\mu \mathrm{mol} / \mathrm{l})$ & $84.4 \pm 17.7$ & $85.5 \pm 18.3$ & $81.3 \pm 16.4$ & 0.487 \\
\hline Blood urea nitrogen (mmol/l) & $9.0 \pm 12.7$ & $10.2 \pm 15.6$ & $6.9 \pm 3.9$ & 0.472 \\
\hline $\operatorname{ALT}(\mathrm{U} / \mathrm{l})$ & $30.1 \pm 15.7$ & $31.8 \pm 17.0$ & $25.0 \pm 10.4$ & 0.268 \\
\hline Total protein (g/l) & $69.1 \pm 8.4$ & $69.3 \pm 7.2$ & $68.6 \pm 11.1$ & 0.853 \\
\hline Albumin (g/l) & $44.0 \pm 6.8$ & $44.1 \pm 6.4$ & $43.9 \pm 8.0$ & 0.948 \\
\hline Sodium (mmol/l) & $140.3 \pm 2.1$ & $140.4 \pm 2.1$ & $140.2 \pm 2.3$ & 0.868 \\
\hline Potassium (mmol/l) & $4.5 \pm 0.4$ & $4.5 \pm 0.3$ & $4.6 \pm 0.5$ & 0.531 \\
\hline Calcium (mmol/l) & $2.3 \pm 0.1$ & $2.3 \pm 0.1$ & $2.3 \pm 0.2$ & 0.689 \\
\hline NT-proBNP (ng/l) & $1328.3 \pm 1420.2$ & $1304.5 \pm 1457.5$ & $1757.2 \pm 1335.2$ & 0.766 \\
\hline
\end{tabular}

ALT alanine transaminase, BMI body mass index, HCM hypertrophic cardiomyopathy, NT-proBNP N-terminal pro-brain natriuretic peptide

Interestingly, the MYBPC3 group had a significantly higher number of patients with a positive family history of HCM ( $46 \%$ vs. $7 \%$; $p=0.014)$.

The most frequently found comorbidity was thyroid gland dysfunction, which was present in 8 patients (13\%) in total, without significant difference between MYBPC3 and MYH7 groups ( $15 \%$ vs. $7 \% ; p=0.422)$. No significant difference between the MYBPC 3 and MYH7 patients was observed in other comorbidities as well: diabetes mellitus $(6 \%$ vs. $0 \%$; $p=0.321)$, chronic obstructive pulmonary disease ( $4 \%$ vs. $0 \%$; $p=0.422)$, anemia ( $2 \%$ vs. $0 \%$; $p=0.573)$.

The mean heart rate was similar between MYBPC3 and MYH7 patients $(64.6 \pm 11.8$ vs. $67.8 \pm 20.4 \mathrm{bpm}$; $p=0.546$ ). However, there was a numerically higher prevalence of atrial fibrillation in the MYH7 group (60\% vs. $35 \%, p=0.085$ ).

Blood laboratory analyses indicating renal and liver function, as well as blood glucose and electrolytes, showed levels within the reference range, without differences between MYBPC3 and MYH7 patients (Table 1).
Levels of N-terminal pro-brain natriuretic peptide (NTproBNP) were elevated in all patients, but without difference among groups.

Echocardiography findings are presented in Table 2 and Fig. 1. There was no difference in the posterolateral wall $(10.6 \pm 2.1$ vs. $10.8 \pm 1.7 \mathrm{~mm}, p=0.776)$ and interventricular septum $(21.5 \pm 7.0$ vs. $21.6 \pm 7.9 \mathrm{~mm}$, $p=0.982)$ thickness between MYBPC3 and MYH7 patients. Left atrial volume was $14 \%$ lower $(p=0.518)$ and left ventricular end-diastolic volume was $19 \%$ higher $(p=0.560)$ in MYBPC3. Left ventricular endsystolic volume was similar between MYBPC3 and MYH7 (52.6 \pm 37.4 vs. $44.0 \pm 19.0 \mathrm{ml} ; p=0.700)$ as was left ventricular ejection fraction $(55.6 \pm 8.2$ vs. $54.1 \pm 6.3, p=0.594)$ and tricuspid annular plane systolic excursion (TAPSE) $(21.0 \pm 4.4$ vs. $22.5 \pm 6.0 \mathrm{~mm}$, $p=0.363)$.

Importantly, the systolic anterior motion was significantly higher in patients carrying MYH7 mutation (33\% vs. $10 \%$; $p=0.025)$, as well as mitral leaflet abnormalities $(40 \%$ vs. $19 \% ; p=0.039)$. Calcifications of mitral 
Table 2 Echocardiography findings in patients with MYBPC3 and MYH7 gene mutation

\begin{tabular}{|c|c|c|c|c|}
\hline & Overall & MYBPC3 & MYH7 & $p$ value \\
\hline PLW thickness (mm) & $10.6 \pm 2.0$ & $10.6 \pm 2.1$ & $10.8 \pm 1.7$ & 0.776 \\
\hline IVS thickness (mm) & $21.5 \pm 7.1$ & $21.5 \pm 7.0$ & $21.6 \pm 7.9$ & 0.982 \\
\hline LA volume (ml) & $115.6 \pm 56$ & $111.7 \pm 83.9$ & $130.3 \pm 87.2$ & 0.518 \\
\hline LV end-diastolic volume (ml) & $108.9 \pm 49.7$ & $110.8 \pm 52.2$ & $92.7 \pm 7.0$ & 0.560 \\
\hline LV end-systolic volume (ml) & $51.6 \pm 35.6$ & $52.6 \pm 37.4$ & $44.0 \pm 19.0$ & 0.700 \\
\hline LV ejection fraction (\%) & $55.3 \pm 7.8$ & $55.6 \pm 8.2$ & $54.1 \pm 6.3$ & 0.594 \\
\hline LV mass (g) & $301.4 \pm 114.0$ & $306.0 \pm 115.2$ & $261.0 \pm 115.4$ & 0.527 \\
\hline LV mass index $\left(\mathrm{g} / \mathrm{m}^{2}\right)$ & $155.9 \pm 52.3$ & $159.0 \pm 53.1$ & $129.5 \pm 43.1$ & 0.364 \\
\hline Relative wall thickness & $0.43 \pm 0.10$ & $0.44 \pm 0.10$ & $0.34 \pm 0.02$ & 0.103 \\
\hline LV outflow pressure gradient $(\mathrm{mmHg})$ & $8.2 \pm 11.1$ & $6.0 \pm 2.5$ & $16.1 \pm 22.7$ & 0.252 \\
\hline E/e' ratio & $9.7 \pm 4.5$ & $8.8 \pm 3.3$ & $13.9 \pm 6.9$ & $0.079^{*}$ \\
\hline TAPSE (mm) & $21.4 \pm 4.8$ & $21.0 \pm 4.4$ & $22.5 \pm 6.0$ & 0.363 \\
\hline Systolic anterior motion, $n(\%)$ & $10(15.9 \%)$ & $5(10.4 \%)$ & $5(33.3 \%)$ & $0.025^{*}$ \\
\hline Papillary muscle abnormalities, n (\%) & $4(6.3 \%)$ & $4(8.3 \%)$ & - & 0.261 \\
\hline Mitral leaflet abnormalities, $n(\%)$ & $15(23.8 \%)$ & $9(18.8 \%)$ & $6(40.0 \%)$ & $0.039^{*}$ \\
\hline Calcification of mitral annulus, $n(\%)$ & $3(4.8 \%)$ & - & $3(20.0 \%)$ & $0.001^{*}$ \\
\hline
\end{tabular}

PLW posterolateral wall, IVS interventricular septum, LA left atrium, LV left ventricle, E/e' LV filling pressure, TAPSE tricuspid annular plane systolic excursion

annulus were registered only in MYH7 patients (20\% vs. $0 \% ; p=0.001$ ).

An interesting finding is the difference of $\mathrm{E} / \mathrm{e}^{\prime}$ ratioa marker of LV filling pressure-between the groups (MYBPC3 8.8 $\pm 3.3, \quad$ MYH7 13.9 $\pm 6.9, \quad p=0.079$ ). Although the level of significance is slightly beyond the threshold, the difference is indicative.

\section{Discussion}

The genetic basis of HCM is more complex than previously thought: known genetic mutations are responsible for about half of the cases, while the remaining causes are unknown. Since variants have not been found to explain the presence of the disease in many patients, there are certainly other, yet unidentified genes. There is an emphasized need to discover additional genetic, epigenetic, and environmental causes that would explain the high proportion of cases of unknown etiology. For many newly reported genes, the lack of strong evidence to support a causal role in HCM creates uncertainty in the interpretation of the results. One of the major roles of genetic testing for HCM patients is better clinical surveillance of asymptomatic family members.

This study analyzed the genetic determinacy of various clinical phenotype parameters among patients with HCM. Only carriers of a single gene mutation, either MYBPC3 or MYH7 were included. Studies that performed genetic screening in large cohorts of patients with a confirmed clinical diagnosis of HCM managed to detect a pathogenic mutation in about $40-50 \%$ of patients $[13,15]$, suggesting that as much as half of the
HCM diagnosed patients do not have known sarcomeric gene mutations.

The MYBPC3 and MYH7 mutations are the two most common mutations among HCM patients with identified sarcomeric gene mutations. A recent meta-analysis on $7675 \mathrm{HCM}$ patients including a total of 51 studies performed by Sedaghat-Hamedani et al. [16] found that the prevalence of MYBPC3 and MYH7 gene mutations were $20 \%$ and $14 \%$, respectively, while all the other mutations had a prevalence below $2 \%$.

$\mathrm{HCM}$ is a disease of a younger age, as it is often first diagnosed before the age of 40 [15, 17]. In our study, patients' mean age was 50 for MYBPC3 and 55 for MYH7 mutations, with no significant difference among groups. This contrasts with previous findings, which suggest earlier onset and diagnosis of the disease for MYH7 mutation $[16,18]$. Patients in our study were predominantly male, which is consistent with gender distribution across literature, where about two-thirds of HCM patients are male $[13,19,20]$.

Olivotto et al. [21] in their multicenter study from 2005, examined differences in HCM presentation among genders in a population of 969 patients. Although most patients were male (59\%), mortality rates did not differ among genders. The authors also pointed out that female patients with HCM although more symptomatic, were under-represented and older. Females were more susceptible to advanced heart failure development, mostly due to LV outflow obstruction. Results from a more recent study from Jang et al. [22] conducted on 202 HCM patients without LV outflow obstruction are in-line with 

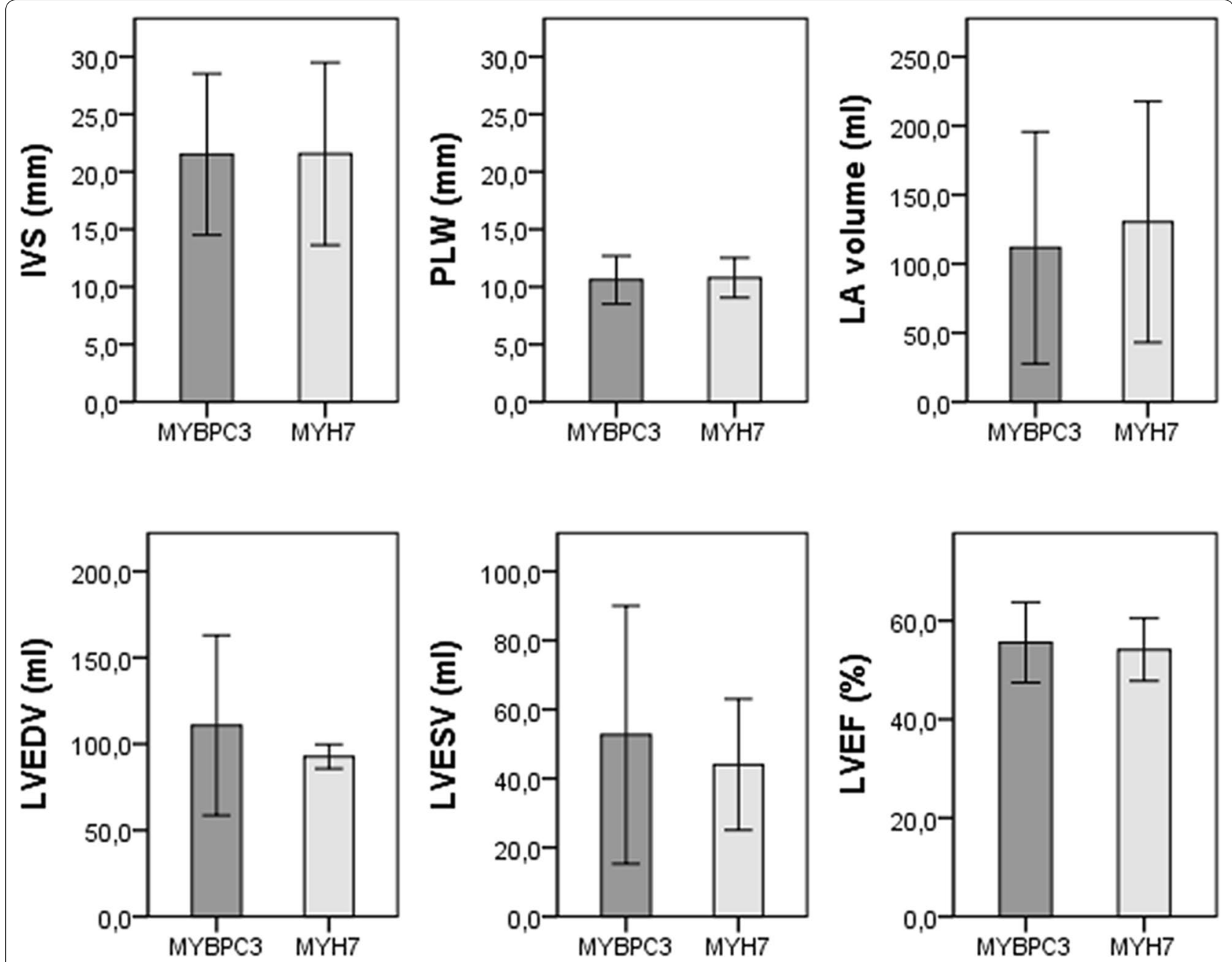

Fig. 1 Echocardiography parameters in MYBPC3 and MYH7 patients (no significant difference was observed in the presented parameters, $p>0.05$ ) (IVS interventricular septum, PLW posterolateral wall, LA left atrium, LVEDV left ventricle end-diastolic volume, LVESV left ventricle end-systolic volume, LVEF left ventricle ejection fraction)

previously mentioned. Jang et al. concluded that females had a higher incidence of heart failure, as well as a greater risk of hospitalization and cardiovascular-related mortality. A higher risk of heart failure in female patients was attributed to the differences in LA and LV morphology and diastolic function between the genders.

Patients with MYBPC3 mutation in our study had a notable number $(46 \%)$ of relatives with a confirmed HCM diagnosis. Across the literature, various rates of positive family history ranging from 25 to $70 \%$ have been reported $[18,19]$. However, the reliability of these numbers should be taken with reserve, because family screening in patients with HCM has still not been fully implemented, despite the clear recommendations for a detailed follow-up of all adult first-degree relatives $[5,23]$. New evidence suggests that screening should be performed even earlier in child age, especially in families with MYBPC3 and MYH7 mutations [24]. Moreover, the diagnosis in relatives is often established solely on phenotypic expression (i.e. imaging methods like echocardiography and cardiac magnetic resonance), without proper genetic testing. Even in the case of performed genetic analysis, currently available methods still fail to identify more than half of patients with HCM [25].

Several studies have attempted to differentiate between disease severity, progression, and phenotype-based on specific mutation subclasses, but there is currently no consensus as to whether a specific phenotype or prognosis can be predicted from an MYBPC3 mutation [26]. Mutation of the MYH7 gene is associated with an earlier onset of symptoms, more pronounced hypertrophy, and poor prognosis [27]. The Arg453Cys mutation of MYH7 is associated with a high incidence of terminal heart 
failure and premature death [28]. Several studies have found a correlation between five mutations (four in the $\mathrm{MYH7}$ gene and one in the gene encoding cardiac troponin $\mathrm{T}$ ) and high incidences of advanced cardiac death, however, these associations were not consistent with the results of other studies [29].

The study by Olivotto et al. [30] assessed the occurrence of atrial fibrillation and outcome in 480 consecutive HCM patients (age at diagnosis, $45 \pm 20$ years; $61 \%$ male) during a follow-up period of $9.1 \pm 6.4$ years. In their cohort, atrial fibrillation was documented in 107 patients, with a prevalence of $22 \%$. The authors concluded that atrial fibrillation is associated with substantial risk for heart failure-related mortality, stroke, and severe functional disability, particularly in patients with outflow obstruction, those $\leq 50$ years of age, or those developing chronic atrial fibrillation.

Atrial fibrillation tended to be more prevalent in the MYH7 group in our study. This finding is consistent with previous studies [17,31], which reported a higher incidence of atrial fibrillation in patients with MYH7 mutation in comparison to other HCM patients. Since the development of atrial fibrillation was associated with risk factors such as LA enlargement, LV wall thickness, and LV outflow tract obstruction, these results suggest that patients with MYH7 mutation present with a more severe clinical phenotype. However, a prospective study on $237 \mathrm{HCM}$ patients with a mean follow-up period of $14 \pm 10$ years found no statistically significant difference in atrial fibrillation between patients with MYBPC3 and MYH7 mutations, with an incidence of $31 \%$ and $37 \%$, respectively [32].

Detailed analysis of echocardiography parameters between the MYBPC3 and MYH7 groups in the present study revealed a somewhat similar phenotype expression with minor differences between the groups, although with slightly more severe disease presentation in the MYH7 group. Most importantly, LV wall hypertrophy was equally expressed in both groups at the posterolateral wall and interventricular septum. Previous studies on larger groups of HCM patients that analyzed myocardial wall thickness measured by both echocardiography $[16-18,33]$ and cardiac magnetic resonance [20] also discovered no significant differences regarding LV wall thickness between MYBPC3 and MYH7 patients. The somewhat counterintuitive finding came from the Florence group [34], stating that LV mass index was normal in about $20 \%$ of patients with definite HCM phenotype and that increased LV mass alone should not be the parameter for establishing the clinical diagnosis of HCM. The LV mass correlated weakly with maximal wall thickness and proved more sensitive in predicting outcomes.
Heart systolic function measured through ejection fraction for LV and TAPSE for right ventricle were preserved in all study patients, with no differences between the groups. This is consistent with previous findings and the current standpoint that HCM generally does not lead to systolic function deterioration. The symptoms and clinical severity are dominantly determined by the combination of diastolic dysfunction, mitral apparatus abnormalities, and LV outflow tract obstruction [35, 36]. A recent study by Miller et al. [37] established that patients with pathogenic, likely pathogenic or rare MYH7 variants had higher LV ejection fraction than those with MYBPC3 variants (68.8 vs. 59.1, $p<0.001)$ and higher right ventricle ejection fraction (67.3 vs. $60.8, p=0.018)$. Additionally, patients with MYBPC3 variants were more likely to have LV ejection fraction $<55 \%(29.7 \%$ vs. $4.9 \%, p=0.005)$.

A very interesting paper from Maron et al. [38] explored mitral valve abnormalities in $\mathrm{HCM}$ patients using cardiovascular magnetic resonance imaging. Mitral valve morphology was observed and compared between 172 patients with $\mathrm{HCM}$ and 172 controls without evidence of cardiovascular disease. After careful characterization, they concluded that mitral valve abnormalities (i.e. leaflet elongation) independently contribute to the severity of HCM presentation, thus expanding the area undesirable effects of HCM genes from solely sarcomere mutations to valvular structures as well. We wanted to further classify mitral valve abnormalities depending on the genetic basis. In this regard, the MYH7 group in our study had a significantly higher number of mitral leaflet abnormalities, mitral annulus calcifications, and the most important higher number of systolic anterior motion, contributing to the worse phenotype expression of MYH7 versus MYBPC3 gene mutations. The study of Groarke et al. [39] observed an increased number of mitral valve abnormalities in patients with sarcomeric gene mutations, however, they did not analyze the difference among the particular gene mutations. Waldmuller et al. [15] on the other hand, reported a more severe level of mitral regurgitation in patients with MYH7 mutation than in patients with MYBPC3 mutation.

Diagnosis of hereditary cardiac disorders based on genetic information is particularly challenging because of the high genetic heterogeneity and overlapping and variable nature of these clinical presentations. The clinical presentation of HCM is influenced by age, lifestyle, and presence of hypertension, among other factors. Although there is still no consensus on the exact impact of gender on HCM presentation and progression, gender influence is thought to exist and that differences in gene expression and hormonal differences affect the symptoms and clinical outcomes of HCM. 
Our study was able to demonstrate the subtle but clinically important difference between patients with different genetic profiles. The clinical implications that may arise from these findings point to the fact that structural abnormalities are more prevalent in $\mathrm{MYH7}$ gene mutation. Patients with MYH7 mutation would probably benefit from more intense imaging surveillance that should start at a younger age as they are likely to develop mitral valve dysfunction and LVOT obstruction. Concerning diastolic dysfunction, it is reasonable to assume that patients with MYH7 gene mutation would benefit from earlier commencement and more aggressive medical treatment. Given the clinical profile, MYH7 mutation patients would be ideal candidates for cardiac myosin inhibitors such as mavacamten. Strenuous exercise should be routinely discouraged, especially in patients with the MYH7 gene mutation.

Our data also suggest and confirm already established management paradigms - an individualized approach concerning specific underlying clinical conditions and pathways (sudden cardiac death risk, heart failure, and atrial fibrillation). Such an approach has been proven to provide the opportunity to aggressively alter the progression of the disease, prevent mortality, and provide normal or extended life expectancy associated with improved quality of life.

\section{Study limitations}

We acknowledge that the large number of operators involved in echocardiographic measurements in this multicenter study represents an unavoidable limitation. However, care was taken to standardize measurements of cardiac dimension and function by prospectively providing detailed technical instructions to all participating centers.

Finally, although the number of included patients in the study is modest, we believe that patient heterogenicity (multicenter study) confers substantial power to our data. Nevertheless, the modest size is one reason to exercise caution in extrapolating these results to the broad spectrum of hypertrophic cardiomyopathy.

A cross-sectional study design does not allow monitoring of disease progression. However, disease progression and response to pharmacological and lifestyle intervention in HCM is subject to our separate ongoing longitudinal SILICOFCM study $[14,40]$.

\section{Conclusions}

Up to this point, numerous mutations leading to HCM have been identified and various clinical manifestations and phenotypic expressions of HCM have been described (from a completely asymptomatic condition, through outflow tract obstruction, diastolic dysfunction, to progressive heart failure and sudden cardiac death). However, no consistent association between the HCM genotype and phenotype have been identified.

In those terms, our study is no exception. Although we focused our attention on the two most common sarcomeric gene mutations responsible for $\mathrm{HCM}-\mathrm{MYBPC} 3$ and MYH7 gene mutations-we were not able to demonstrate any substantial differences regarding clinical and echocardiography findings. More frequent systolic anterior motion and other mitral valve abnormalities as well as increased left ventricle filling pressure in $\mathrm{MYH7}$ gene mutation suggests that MYH7 gene mutation does present with a more severe disease phenotype.

Correlation between the genetic and clinical status of HCM patients remains elusive in most of the cases-limitation with a major impact on the development of personalized medicine approaches. Our study might subtly add to the overall understanding of such complex relations and might push genetic testing results from strictly diagnostic to prognostic fashion.

\section{Abbreviations \\ ACTC: a-Actin; BMI: Body mass index; BSA: Body surface area; CO: CardiaC output; E/e': Left ventricle filling pressure; HCM: Hypertrophic cardiomyo- pathy; HR: Heart rate; IVS: Interventricular septum; LA: Left atrium; LV: Left ventricle; LVEDV: Left ventricular end-diastolic volume; LVESV: Left ventricle end-systolic volume; LVmass: Left ventricle myocardial mass; MYBPC3: Myosin- binding protein C; MYH7: $\beta-$ Myosin heavy chain; PLW: Posterolateral wall; RWT : Relative wall thickness; SV: Stroke volume; TAPSE: Tricuspid annular plane systolic excursion; TNNI3: Troponin I type 3; TNNT2: Troponin T type 2; TPM1: a-Tropomyosin.}

\section{Acknowledgements}

Part of this investigation was presented in the form of a poster presentation at the EuroHeartCare ACNAP Congress 2020.

\section{Authors' contributions}

LV, DGJ, LM, and IO conceptualized and designed the study. LV and AP analyzed and interpreted the data, and wrote the manuscript. LV, DGJ, AR, GAM, $L M$, and $I O$ revised the manuscript. AP, MG, MB, AI, SS, FB, MT, NO, MT, PB, NF, and DP participated in data acquisition and database creation. All authors read and approved the final manuscript.

\section{Funding}

This work has been conducted as part of the SILICOFCM project which received funding from the European Union's Horizon 2020 Research and Innovation Programme under Grant Agreement No. 777204. The funder did not influence the design of the study and data collection, analysis, and interpretation of data, nor writing the manuscript.

\section{Availability of data and materials}

The datasets used and analyzed during the current study are available from the corresponding author on reasonable request.

\section{Ethics approval and consent to participate}

The study was approved by the UK National Health Service Health Research Authority North East-Tyne \& Wear South Research Ethics Committee with reference number 18/NE/0318 and adopted by the Institutional Review Board of each participating institution with study participants providing written informed consent.

Consent for publication

Not applicable. 


\section{Competing interests}

The authors declare that they have no competing interests.

\section{Author details}

${ }^{1}$ Faculty of Medicine, University of Novi Sad, Novi Sad, Serbia. ${ }^{2}$ Institute of Cardiovascular Diseases of Vojvodina, Sremska Kamenica, Serbia. ${ }^{3}$ Cardiovascular Research, Translational and Clinical Research Institute, Medicine, Newcastle University, Newcastle upon Tyne Hospitals NHS Foundation Trust, Newcastle upon Tyne, UK. ${ }^{4}$ Careggi University Hospital, University of Florence, Florence, Italy. ${ }^{5}$ Department of Internal Medicine II (Cardiology, Pneumology, and Intensive Care), University Medical Centre Regensburg, Regensburg, Germany. ${ }^{6}$ Cardiology Department, Clinical Centre of Serbia, Faculties of Medicine and Pharmacy, University of Belgrade, Belgrade, Serbia. ${ }^{7}$ Bioengineering Research and Development Center, BioIRC, Kragujevac, Serbia. ${ }^{8}$ Faculty of Engineering, University of Kragujevac, Kragujevac, Serbia. ${ }^{9}$ Faculty of Health and Life Sciences, Coventry University, and University Hospitals Coventry and Warwickshire NHS Trust, Coventry, UK.

Received: 17 June 2020 Accepted: 2 December 2020

Published online: 09 December 2020

\section{References}

1. Maron BJ, Maron MS. Hypertrophic cardiomyopathy. Lancet. 2013;381(9862):242-55.

2. Maron BJ. Clinical course and management of hypertrophic cardiomyopathy. N Engl J Med. 2018;379(20):1977.

3. Gersh BJ, Maron BJ, Bonow RO, Dearani JA, Fifer MA, Link MS, et al. 2011 ACCF/AHA guideline for the diagnosis and treatment of hypertrophic cardiomyopathy: executive summary: a report of the American College of Cardiology Foundation/American Heart Association Task Force on Practice Guidelines. Circulation. 2011a;124(24):2761-96.

4. Maron BJ, Ommen SR, Semsarian C, Spirito P, Olivotto I, Maron MS. Hypertrophic cardiomyopathy: present and future, with translation into contemporary cardiovascular medicine. J Am Coll Cardiol. 2014;64(1):83-99.

5. Authors/Task Force Members, Elliott PM, Anastasakis A, Borger MA, Borg grefe M, Cecchi F, et al. 2014 ESC Guidelines on diagnosis and management of hypertrophic cardiomyopathy: the Task Force for the Diagnosis and Management of Hypertrophic Cardiomyopathy of the European Society of Cardiology (ESC). Eur Heart J. 2014;35(39):2733-79.

6. Force T, Bonow RO, Houser SR, Solaro RJ, Hershberger RE, Adhikari B, et al. Research priorities in hypertrophic cardiomyopathy: report of a Working Group of the National Heart, Lung, and Blood Institute. Circulation. 2010;122(11):1130-3.

7. Lu DY, Pozios I, Haileselassie B, Ventoulis I, Liu H, Sorensen LL, et al. Clinical outcomes in patients with nonobstructive, labile, and obstructive hypertrophic cardiomyopathy. J Am Heart Assoc. 2018;7(5):e006657.

8. Lopes LR, Rahman MS, Elliott PM. A systematic review and meta-analysis of genotype-phenotype associations in patients with hypertrophic cardiomyopathy caused by sarcomeric protein mutations. Heart. 2013;99(24):1800-11.

9. Garfinkel AC, Seidman JG, Seidman CE. Genetic pathogenesis of hypertrophic and dilated cardiomyopathy. Heart Fail Clin. 2018;14(2):139-46.

10. Olivotto I, Girolami F, Ackerman MJ, Nistri S, Bos JM, Zachara E, et al. Myofilament protein gene mutation screening and outcome of patients with hypertrophic cardiomyopathy. Mayo Clin Proc. 2008;83(6):630-8.

11. Keren A, Syrris P, McKenna WJ. Hypertrophic cardiomyopathy: the genetic determinants of clinical disease expression. Nat Clin Pract Cardiovasc Med. 2008;5(3):158-68.

12. Mazzarotto F, Girolami F, Boschi B, Barlocco F, Tomberli A, Baldini K, et al. Defining the diagnostic effectiveness of genes for inclusion in panels: the experience of two decades of genetic testing for hypertrophic cardiomyopathy at a single center. Genet Med. 2019;21(2):284-92.

13. Millat G, Bouvagnet $P$, Chevalier P, Dauphin C, Jouk PS, Da Costa A, et al. Prevalence and spectrum of mutations in a cohort of 192 unrelated patients with hypertrophic cardiomyopathy. Eur J Med Genet. 2010;53(5):261-7.

14. Tafelmeier M, Baessler A, Wagner S, Unsoeld B, Preveden A, Barlocco F, et al. Design of the SILICOFCM study: effect of sacubitril/valsartan vs lifestyle intervention on functional capacity in patients with hypertrophic cardiomyopathy. Clin Cardiol. 2020;43:430-40.

15. Waldmuller S, Erdmann J, Binner P, Gelbrich G, Pankuweit S, Geier C, et al. Novel correlations between the genotype and the phenotype of hypertrophic and dilated cardiomyopathy: results from the German Competence Network Heart Failure. Eur J Heart Fail. 2011;13(11):1185-92.

16. Sedaghat-Hamedani F, Kayvanpour E, Tugrul OF, Lai A, Amr A, Haas J, et al. Clinical outcomes associated with sarcomere mutations in hypertrophic cardiomyopathy: a meta-analysis on 7675 individuals. Clin Res Cardiol. 2018;107(1):30-41.

17. Marsiglia JD, Credidio FL, de Oliveira TG, Reis RF, Antunes Mde O, de Araujo AQ, et al. Screening of MYH7, MYBPC3, and TNNT2 genes in Brazilian patients with hypertrophic cardiomyopathy. Am Heart J. 2013;166(4):775-82.

18. Garcia-Castro M, Coto E, Reguero JR, Berrazueta JR, Alvarez V, Alonso B, et al. Mutations in sarcomeric genes MYH7, MYBPC3, TNNT2, TNNI3, and TPM1 in patients with hypertrophic cardiomyopathy. Rev Esp Cardiol. 2009;62(1):48-56.

19. Liu X, Jiang T, Piao C, Li X, Guo J, Zheng S, et al. Screening mutations of MYBPC3 in 114 unrelated patients with hypertrophic cardiomyopathy by targeted capture and next-generation sequencing. Sci Rep. 2015;5:11411.

20. Weissler-Snir A, Hindieh W, Gruner C, Fourey D, Appelbaum E, Rowin $E$, et al. Lack of phenotypic differences by cardiovascular magnetic resonance imaging in MYH7 (beta-myosin heavy chain)-versus MYBPC3 (myosin-binding protein C)-related hypertrophic cardiomyopathy. Circ Cardiovasc imaging. 2017;10(2):e005311.

21. Olivotto I, Maron MS, Adabag AS, Casey SA, Vargiu D, Link MS, et al. Gender-related differences in the clinical presentation and outcome of hypertrophic cardiomyopathy. J Am Coll Cardiol. 2005;46(3):480-7.

22. Jang JH, Shin SH, Beak YS, Ko KY, Kwon SW, Park SD, et al. Impact of gender on heart failure presentation in non-obstructive hypertrophic cardiomyopathy. Heart Vessels. 2020;35(2):214-22.

23. Gersh BJ, Maron BJ, Bonow RO, Dearani JA, Fifer MA, Link MS, et al. 2011 ACCF/AHA guideline for the diagnosis and treatment of hypertrophic cardiomyopathy: a report of the American College of Cardiology Foundation/American Heart Association Task Force on Practice Guidelines. Circulation. 2011b;124(24):e783-831.

24. Semsarian C, Ho CY. Screening children at risk for hypertrophic cardiomyopathy: balancing benefits and harms. Eur Heart J. 2019;40(45):3682-4.

25. Marian AJ, Braunwald E. Hypertrophic cardiomyopathy: genetics, pathogenesis, clinical manifestations, diagnosis, and therapy. Circ Res. 2017;121(7):749-70.

26. Kraft T, Montag J, Radocaj A, Brenner B. Hypertrophic cardiomyopathy: cell-to-cell imbalance in gene expression and contraction force as trigger for disease phenotype development. Circ Res. 2016;119(9):992-5.

27. Hallioglu Kilinc O, Giray D, Bisgin A, Tug Bozdogan S, Karpuz D. Familial hypertrophic cardiomyopathy: a case with a new mutation in the MYBPC3 gene. Turk Kardiyoloji Dernegi arsivi Turk Kardiyoloji Derneginin yayin organidir. 2017;45(5):450-3.

28. Tower-Rader A, Desai MY. Phenotype-genotype correlation in hypertrophic cardiomyopathy: less signal, more noise? Circ Cardiovasc Imaging. 2017;10(2):e006066.

29. Weissler-Snir A, Adler A, Williams L, Gruner C, Rakowski H. Prevention of sudden death in hypertrophic cardiomyopathy: bridging the gaps in knowledge. Eur Heart J. 2017;38(22):1728-37.

30. Olivotto I, Cecchi F, Casey SA, Dolara A, Traverse JH, Maron BJ. Impact of atrial fibrillation on the clinical course of hypertrophic cardiomyopathy. Circulation. 2001;104(21):2517-24.

31. Lee SP, Ashley EA, Homburger J, Caleshu C, Green EM, Jacoby D, et al. Incident atrial fibrillation is associated with MYH7 sarcomeric gene variation in hypertrophic cardiomyopathy. Circ Heart Fail. 2018;11(9):e005191.

32. Bongini C, Ferrantini C, Girolami F, Coppini R, Arretini A, Targetti M, et al. Impact of genotype on the occurrence of atrial fibrillation in patients with hypertrophic cardiomyopathy. Am J Cardiol. 2016;117(7):1151-9.

33. Viswanathan SK, Sanders HK, McNamara JW, Jagadeesan A, Jahangir A, Tajik AJ, et al. Hypertrophic cardiomyopathy clinical phenotype is independent of gene mutation and mutation dosage. PLoS ONE. 2017;12(11):e0187948.

34. Olivotto I, Maron MS, Autore C, Lesser JR, Rega L, Casolo G, et al. Assessment and significance of left ventricular mass by cardiovascular 
magnetic resonance in hypertrophic cardiomyopathy. J Am Coll Cardiol. 2008;52(7):559-66.

35. Olivotto I, Cecchi F, Poggesi C, Yacoub MH. Patterns of disease progression in hypertrophic cardiomyopathy: an individualized approach to clinical staging. Circ Heart Fail. 2012;5(4):535-46.

36. Ommen SR, Shah PM, Tajik AJ. Left ventricular outflow tract obstruction in hypertrophic cardiomyopathy: past, present and future. Heart. 2008;94(10):1276-81.

37. Miller RJH, Heidary S, Pavlovic A, Schlachter A, Dash R, Fleischmann $D$, et al. Defining genotype-phenotype relationships in patients with hypertrophic cardiomyopathy using cardiovascular magnetic resonance imaging. PLoS ONE. 2019;14(6):e0217612.

38. Maron MS, Olivotto I, Harrigan C, Appelbaum E, Gibson CM, Lesser JR, et al. Mitral valve abnormalities identified by cardiovascular magnetic resonance represent a primary phenotypic expression of hypertrophic cardiomyopathy. Circulation. 2011;124(1):40-7.
39. Groarke JD, Galazka PZ, Cirino AL, Lakdawala NK, Thune JJ, Bundgaard $\mathrm{H}$, et al. Intrinsic mitral valve alterations in hypertrophic cardiomyopathy sarcomere mutation carriers. Eur Heart J Cardiovasc Imaging. 2018;19(10):1109-16.

40. Velicki L, Preveden A, Tafelmeier M, Olivotto I, Barlocco F, Popovic D, et al. Genetic determinants of clinical phenotype in hypertrophic cardiomyopathy. Eur J Cardiovasc Nurs. 2020;19(1_suppl):S43-4.

\section{Publisher's Note}

Springer Nature remains neutral with regard to jurisdictional claims in published maps and institutional affiliations.
Ready to submit your research? Choose BMC and benefit from:

- fast, convenient online submission

- thorough peer review by experienced researchers in your field

- rapid publication on acceptance

- support for research data, including large and complex data types

- gold Open Access which fosters wider collaboration and increased citations

- maximum visibility for your research: over $100 \mathrm{M}$ website views per year

At BMC, research is always in progress.

Learn more biomedcentral.com/submissions 